tolerated. These results indicate that probiotic supplementation confers benefit in IBS-D and deserves further investigation.

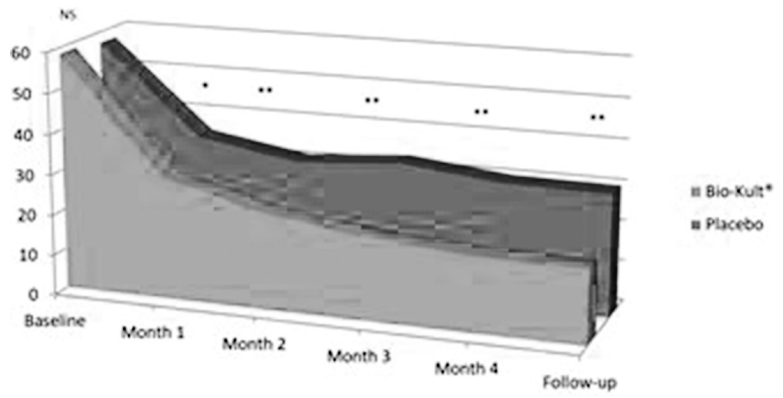

Abstract OWE-032 Figure 1 lbs-sss abdominal pain rating with probiotic (bio kult $(\mathrm{X})$ or placebo(16 weeks treatment and1-month follow up

The lower the score less the pain: $* \mathrm{p} \geq 0.002 * \mathrm{p} \leq 0.001$ : NS $\geq$ Not significant

\section{PWE-127 GASTROINTESTINAL DYSMOTILITY - THE CONUNDRUM OF FEEDING: A NEW TECHNIQUE}

Rebecca Anderson*, Simon Higgs, Steve Hornby, Richard Makins. Gloucestershire Hospitals NHS Foundation Trust, Cheltenham, UK

\subsection{6/gutjnl-2018-BSGAbstracts.421}

Introduction Gastrointestinal (GI) dysmotility disorders can be debilitating and their management challenging. Patients often require feeding tubes to support nutrition, which can be complicated by discomfort and/or displacement. Laparoscopically Assisted-Enteroscopically Positioned Jejunostomy Tube (LAEPJT) insertion is a novel technique not previously described in GI literature. It involves enteroscopic insertion of a jejunostomy tube under direct laparoscopic vision and general anaesthesia. This study reviews the experience of LA-EPJT insertion for GI dysmotility disorders at a district hospital.

Methods Endoscopy records were used to identify all patients who had undergone LA-EPJT insertion. Patient notes were reviewed to identify indications, previous tube requirements and outcomes.

Results 7 LA-EPJTs were placed between 2014 and 2017 (6 females; age 19-77; median 35; mean 43). Table 1 shows patient symptoms and indications for LA-EPJT insertion.

No tubes migrated or were dislodged after insertion. 1/7 patients $(14.3 \%)$ had early bleeding and tube-site infection. Perforation rates were $0 \%$. $5 / 7(71.4 \%)$ had initial pain with feeding after LA-EPJT insertion although this universally subsided, 1/7 (14.3\%) had ongoing vomiting. 5/7 (71.4\%) tubes remain in situ. 1 was replaced with a PEG-J to allow venting of intestinal contents, and 1 was removed and TPN commenced (psychological factors precluded continued tubefeeding).

Conclusions This study suggests that LA-EPJTs are useful nutritional adjuncts for patients with GI dysmotility disorders. They are a more permanent solution, with little risk of migration. They appear to better control symptoms such as pain and vomiting than traditional tubes, and are simple to change. The procedure benefits from direct laparoscopic vision of the jejunum and therefore carries low perforation rates.

Further studies are needed to assess long-term complications and efficacy of LA-EPJTs for symptom control and nutrition.
However, this data is promising and GI centres could consider adoption of this novel technique for patients with GI dysmotility disorders.

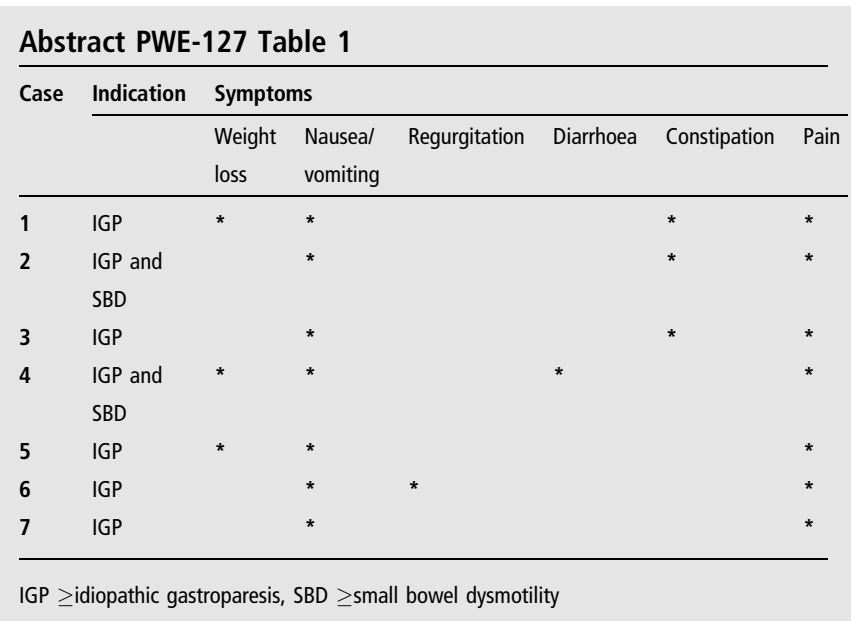

Previous non-pharmacological treatments and nutritional support utilised by patients are shown in table 2 .

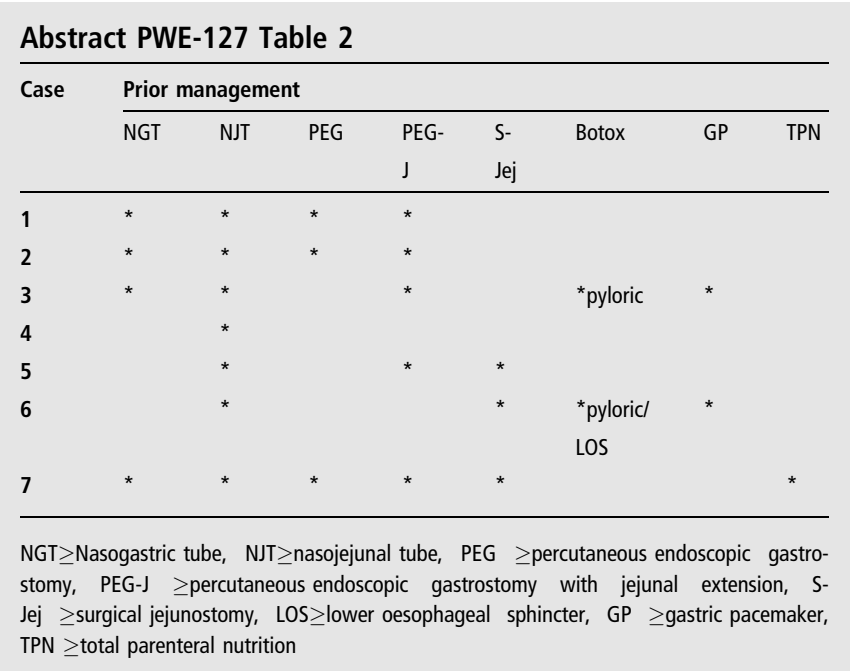

\section{PWE-128 HYPNOTHERAPY FOR IRRITABLE BOWEL SYNDROME: THE PATIENT'S PERCEPTION}

Anne-sophie Donnet*, Shariq Hasan, Vivien Miller, Peter Whorwell. Wythenshawe Hospital, Manchester, UK

\subsection{6/gutjnl-2018-BSGAbstracts.422}

Background Numerous studies have shown that hypnotherapy (HT) improves the symptoms of irritable bowel syndrome (IBS) using clinical outcome measures. In light of the increasing interest in capturing the patient's perception of their illness and treatment, it was felt it would be helpful to record how patients perceive the hypnotherapeutic process, on which there is currently little information.

Aims In addition to measuring symptom change, we have recently started to record the patient's perception of hypnotherapy for their IBS, including their expectations, and now report the results for the first 50 patients.

Methods 50 consecutive IBS patients (38 females and 12 males, age range 18-76) attending for hypnotherapy were 